\title{
Effect of Different Methods and Levels of Zinc and Boron Application on Growth and Yield of Rice under Acid Soils of Hilly Zone of Karnataka
}

\author{
N.R. Ravikiran, S. Channakeshava", R. Jayaramaiah and A. Nataraja \\ College of Agriculture, Karekere, Hassan, University of Agricultural Sciences, \\ Bengaluru, Karnataka, India \\ *Corresponding author
}

\begin{tabular}{|l|}
\hline Ke y w or d s \\
Zinc, Boron, \\
Nutrient content, \\
Grain yield, Straw \\
yield
\end{tabular}

A B S T R A C T

A field experiment was carried out to study the effect of different methods and levels of zinc and boron application on growth and yield of rice under acid soils of hilly zone of Karnataka during Kharif season of 2017 at Sakaleshpura. The experiment consist of 14 treatments replicated thrice using RCBD. The results revealed that significantly higher growth and yield parameters such as plant height $(108.8 \mathrm{~cm})$, number of tillers per hill (20.6), number of panicles (103.5), grain $(64.4 \mathrm{q} / \mathrm{ha})$ and straw $(89.4 \mathrm{q} / \mathrm{ha}$ ) were recorded in treatment receiving foliar spray of zinc sulphate @ 0.5 percent and solubor @ 0.25 percent along with RDF+ FYM as compared RDF+ FYM only. Significantly higher nitrogen $(1.21 \& 1.37 \%)$, phosphorus $(0.34 \% \& 0.49 \%)$, potassium $(0.62$ and $0.51 \%)$, calcium $(0.85 \& 0.53 \%)$ magnesium $(0.24 \& 0.30 \%)$ and sulphur $(0.25 \& 0.30$ $\%)$ in grain and straw respectively. The lowest nutrient content in grain and straw was recorded in RDF + FYM. The concentration of micronutrients differs significantly due to different treatments. However, significantly higher concentration of zinc ( $44.59 \&$ $37.13 \mathrm{mg} / \mathrm{kg}$ ) and boron $(2.15 \& 3.41 \mathrm{mg} / \mathrm{kg})$ in straw and grain at harvest significantly increased due to foliar application of zinc and boron @ 0.5 percent and 0.25 percent respectively. Lowest concentration of zinc $(25.04 \& 21.50 \mathrm{mg} / \mathrm{kg})$ and boron $(1.15 \& 1.89 \mathrm{mg} / \mathrm{kg})$ recorded in RDF+ FYM in straw and grain at harvest

\section{Introduction}

Rice is a staple and major food crop of India. Asia's rice production mainly depends on irrigated rice fields which produce three fourth of all rice harvested. In India, rice is being grown in an area of 433.88 lakh ha with annual production of $104.32 \mathrm{~m} \mathrm{t}$ and productivity of $2404 \mathrm{~kg} \mathrm{ha}^{-1}$ (Anon., 2016a). In Karnataka, rice is being grown in an area of
14 lakh ha with annual production of 39.55 lakh tons and productivity of $2700 \mathrm{~kg} \mathrm{ha}^{-1}$ (Anon., 2016b).

Karnataka has diverse type of soils and climate which is suitable for cultivation of varieties of crops. Application of only N, P and $\mathrm{K}$ fertilizers and growing of high yielding varieties and hybrids are the factors responsible for micronutrient deficiency in 
soil during green revolution period in India. Zinc is necessary for plant metabolism including plant enzymatic activities, production of carbohydrates, proteins auxins and reproduction processes. Zinc is required for biosynthesis of plant growth regulator such as indole 3 acetic acid (IAA) (Fang et al., 2008). Zinc may be found in exchange sites of clay minerals and organic matter or adsorbed on solid surfaces. The zinc availability decreases with increase in $\mathrm{pH}$ of soil. The increased soil $\mathrm{pH}$ causes precipitation of zinc as insoluble amorphous soil zinc and $\mathrm{Zn} \mathrm{SiO}_{4}$. Zinc deficiency can be observed in leaves as visual symptoms. In rice zinc deficiency is called as khaira disease where in plants show white patches on foliage and become stunted in growth. The concentration of zinc in plants is a function of available zinc in soil.

Hence application of zinc fertilizer is essential in keeping sufficient amount of available zinc in soil solution maintaining adequate zinc transport to seeds and for increase in crop yield. There are many ways to apply zinc to crop such as soil application, foliar spray seed treatment fertigation etc. Foliar or combined soil and foliar application under field conditions proved to be highly effective and can be practical way to maximise the zinc accumulation and uptake in grains (Cakmak, 2008).

Boron responsible for better pollination seed setting and grain formation in different rice varieties (Aslam et al., 2002; Rehman et al., 2012) making it more important during reproductive stage as compared to vegetative stage. Boron is needed in small amount but proved to be an essential micronutrient for plant growth. Boron availability to plants decreases under high rainfall and strongly acid soil ( $\mathrm{pH}$ less than 5.0) because boron sorption to iron and aluminium oxide surfaces of soil minerals. Boron application improve plant functioning at cellular level including cell division, flower and fruit formation, carbohydrate and nitrogen metabolism and disease resistance. Boron can be used as soil and foliar application to crop. Foliar application of Boron believed to retain significant carbohydrate phloem mobility to flowering meristematic cell from either senescing leaves and or bark. Rice needs seventeen essential nutrients which supplied through soil in the form of manures and fertilizers.

Micronutrient deficiency such as zinc and boron is wide spread in rice growing area of country that leads to substantial loss in yield and quality of grain with respect to protein and fat content. In addition to recommended dosage of NPK addition of Zinc and boron needed to increase the growth and yield of rice and nutrient uptake. Hence an investigation on Effect of different levels and methods of application of zinc and boron on growth and yield of rice under acid soils in hilly zone of Karnataka

\section{Materials and Methods}

A field experiment was conducted to study the effect of different levels and methods of application of Zinc and boron on growth and yield rice under acid soils in hilly zone of Karnataka at Sakaleshpura, Hassan district during 2017-18. The soil properties of experimental site was analysed using standard procedure (Jackson, 1973) and the details of physicochemical properties given in Table 1 . The experiment was layed out with 14 treatments replicated thrice in Randomized Block design. The plot size was 12 square meters. The rice variety Tunga was transplanted with spacing of $20 \times 10 \mathrm{~cm}$. The fertilizer applied as per the recommended dosage (100:50:50 NPK kg/ha), at the time of transplanting 50\% Nitrogen and 100 Percent Phosphorus and 50 percent Potassium along with Zinc and Boron as per the treatment 
details applied. The crop was top dressed with 25 percent recommended Nitrogen after 40 days of planting and at panicle stage 25 percent $\mathrm{N}$ and 50 percent recommended $\mathrm{K}$ applied. Foliar spray of Zinc and boron given as per the treatments and plant protections has been taken for blast using carbendazine@ 1.0 gper litre and stem borer managed with chloropyriphos@2.0 ml/litre.

\section{Treatment details}

T1: RDF+ FYM, T2: RDF + Soil application of Zn SO4 @ $10 \mathrm{~kg} / \mathrm{ha}, \mathrm{T} 3: \mathrm{RDF}+$ Soil application of Zn SO4 @ 20 kg/ha, T4: RDF + Soil application of Borax @ $2.5 \mathrm{~kg} / \mathrm{ha}, \mathrm{T} 5$ : $\mathrm{RDF}+$ Soil application of Borax @ 5.0 $\mathrm{kg} / \mathrm{ha}$,T6: RDF + Soil application of Zn SO4 @ $10 \mathrm{~kg} / \mathrm{ha}+$ Soil application of Borax @ 2.5 $\mathrm{kg} / \mathrm{ha}$, T7: RDF + Soil application of Zn SO4 @ $20 \mathrm{~kg} / \mathrm{ha}+$ Soil application of Borax @ 5.0 $\mathrm{kg} / \mathrm{ha}$, T8: RDF + Soil application of Zn SO4 @ $20 \mathrm{~kg} / \mathrm{ha}+$ Soil application of Borax @ 2.5 $\mathrm{kg} / \mathrm{ha}$, T9: RDF + Soil application of Zn SO4 @ $20 \mathrm{~kg} / \mathrm{ha}+$ Soil application of Borax @ 5.0 kg/ha, T10: RDF + Foliar spray of Zn SO4 @ $0.5 \%, \mathrm{~T} 11: \mathrm{RDF}+$ Foliar spray of solubor @ $0.1 \%, \mathrm{~T} 12: \mathrm{RDF}+$ Foliar spray of solubor @ $0.25 \%$ T13: RDF + Foliar spray of Zn SO4 @ $0.5 \%$ + Foliar spray of solubor @ $0.1 \%$, T14: RDF + Foliar spray of Zn SO4 @ $0.5 \%+$ Foliar spray of solubor @ 0.25\%.Irrigation and Weed management has been taken as per package of practice. Zinc sprayed at 20 Days after planting and Boron sprayed at 45 days after transplanting.

The data on growth (plant height, number of tillers/hill and number of leaves) and yield parameters (Number of panicles/hill, panicle length, number of filled grains per panicle and test weight) collected at different growth stages $(30,60 \&$ harvest) from all the plots. The soil samples collected at harvest from all the treatments were analysed for major, secondary and micronutrients. The data on growth, yield parameters, soil nutrient status and their uptake by rice crop were analysed statistically using standard procedure (Gomez and Gomez 1984).

\section{Results and Discussion}

The results on effect of different levels and methods of zinc and boron application on growth and yield of rice presented with discussion in paragraph as below (Table 1-6).

\section{Growth parameters}

Results from the data reveals that growth parameters like plant height, number of tillers per hill and number of leaves per hill differ significantly due to different levels and methods of application of zinc and boron. Significantly higher plant height $(108.8 \mathrm{~cm})$, number of tillers/hill (20.6) and number of leaves per hill (59.7) was recorded due to foliar spray of $\mathrm{Zn} \mathrm{SO} 4$ @ 0.5 percent and boron@ 0.25 percent along with RDF and was on par with soil application of Zn SO4 @ $10 \mathrm{~kg} / \mathrm{ha}$ and $20 \mathrm{~kg} / \mathrm{ha}$ and boron @ $2.5 \mathrm{~kg} / \mathrm{ha}$ and $5.0 \mathrm{~kg} / \mathrm{ha}$ respectively. However was on par with T9 and T13 treatments.

The lowest plant height $(89.33 \mathrm{~cm})$, number of tillers per hill (12.6) and number of leaves (40.33) recorded in RDF + FYM. Increase in growth parameters due to $\mathrm{Zn}$ and $\mathrm{B}$ application may be due to enhanced photosynthetic and metabolic activity which lead to an increase in various plant metabolic pathways responsible for cell division and elongation (Chaudhary et al., 2014) resulting in production of new tissues. The acceleration in photosynthesis is evident in better vegetative growth. These findings are in conformity with those of Arif et al., (2012) they have reported that foliar application of $\mathrm{Zn}$ and $\mathrm{B}$ at the rate of $6 \mathrm{~kg}$ acre ${ }^{-1}$ and $3 \mathrm{~kg}$ acre $^{-1}$ recorded 38.40 per cent increase in productive tillers per plant compared to the RDF+FYM. 


\section{Yield parameters}

Data on Yield parameters differ significantly due to soil and foliar application of zinc and boron along with NPK. Significantly higher number of panicles per hill (16.73), panicle length $(28.68 \mathrm{~cm})$ and number of grains per panicles (120.70), number of filled grains per panicles (103.5) and test weight $(3.47 \mathrm{~g})$ recorded due to foliar spray of $\mathrm{Zn} \mathrm{SO} 4$ @ 0.5 percent and solubor @ 0.25 percent along with RDF and however was on par with T13 which received foliar spray of Zn SO4 @ 0.5 percent and solubor@0.1 percent along with RDF. Lowest number of panicles (10.20), panicle length $(18.16 \mathrm{~cm})$, number of grains $(96.86)$ and number of filled grains per panicle (74.53) and test weight $(25.24 \mathrm{~g})$ recorded in $\mathrm{RDF}+$ FYM.

\section{Grain and Straw yield}

The data on grain and straw yield indicated that significantly higher grain $(64.40 \mathrm{q} / \mathrm{ha})$ and straw yield $(89.40 \mathrm{q} / \mathrm{ha})$ was recorded due to foliar spray of zinc and boron @ 0.5 percent and 0.25 percent respectively. However was on par with soil application of zinc sulphate and borax @ $20 \mathrm{~kg} / \mathrm{ha}$ and $5.0 \mathrm{~kg} / \mathrm{ha}$ respectively (61.48 \& 84.693q/ha). The lowest grain and straw yield was recorded with NPK + FYM (48.78 \& 61.86q/ha).

Table.1 Initial physico-chemical properties of experimental site

\begin{tabular}{|c|c|c|}
\hline SL NO & Parameters & Value \\
\hline \multicolumn{3}{|c|}{ Physical properties } \\
\hline 01 & Sand $\%$ & 54.73 \\
\hline 02 & Silt $\%$ & 24.12 \\
\hline $\mathbf{0 3}$ & Clay $\%$ & 20.30 \\
\hline 04 & Textural Class & Sandy clay loam \\
\hline \multicolumn{3}{|c|}{ Chemical properties } \\
\hline 01 & $\mathrm{pH}$ & 4.17 \\
\hline 02 & $\mathrm{EC}(\mathrm{dS} / \mathrm{m})$ & 0.09 \\
\hline 03 & Organic carbon $(\%)$ & 8.10 \\
\hline 04 & Available N(kg/ha) & 250.85 \\
\hline 05 & Available P2 O5 (kg/ha) & 25.19 \\
\hline 06 & Available $\mathrm{K} 2 \mathrm{O}$ (kg/ha) & 69.64 \\
\hline 07 & Available $\mathrm{Ca}(\mathrm{cmol} / \mathrm{kg})$ & 1.50 \\
\hline 08 & Available $\mathrm{Mg}(\mathrm{cmol} / \mathrm{kg})$ & 0.40 \\
\hline 09 & Available $\mathrm{S}(\mathrm{mg} / \mathrm{kg})$ & 20.00 \\
\hline 10 & DTPA Fe & 11.60 \\
\hline 11 & DTPA Zn & 1.58 \\
\hline 12 & DTPA Mn & 6.50 \\
\hline 13 & DTPA Cu & 2.51 \\
\hline 14 & Hot water soluble boron & 0.20 \\
\hline
\end{tabular}


Table.2 Effect of different methods and levels of zinc and boron application on growth and yield parameters of rice

\begin{tabular}{|c|c|c|c|c|c|c|}
\hline $\begin{array}{l}\text { Sl. } \\
\text { No }\end{array}$ & Treatments & $\begin{array}{l}\text { Plant } \\
\text { height } \\
(\mathrm{cm})\end{array}$ & $\begin{array}{l}\text { Number } \\
\text { of tillers } \\
\text { per hill }\end{array}$ & $\begin{array}{c}\text { Number } \\
\text { of } \\
\text { leaves } \\
\text { per hill }\end{array}$ & $\begin{array}{c}\begin{array}{c}\text { Number } \\
\text { of } \\
\text { panicles } \\
\text { per hill }\end{array} \\
\end{array}$ & $\begin{array}{c}\text { Panicle } \\
\text { length } \\
(\mathbf{c m})\end{array}$ \\
\hline & & \multicolumn{5}{|c|}{ Harvest } \\
\hline $\mathrm{T}_{1}$ & RDF +FYM & 89.33 & 12.60 & 40.33 & 10.20 & 18.16 \\
\hline $\mathrm{T}_{2}$ & $\begin{array}{l}\mathrm{T}_{1}+\text { soil application of zinc } \\
\text { sulphate@ } 10 \mathrm{~kg} \mathrm{ha}^{-1}\end{array}$ & 95.40 & 13.63 & 45.20 & 11.30 & 21.52 \\
\hline $\mathrm{T}_{3}$ & $\begin{array}{l}\mathrm{T}_{1}+\text { soil application of zinc } \\
\text { sulphate@20 kg ha- }\end{array}$ & 96.53 & 14.60 & 47.80 & 11.96 & 21.83 \\
\hline $\mathrm{T}_{4}$ & $\begin{array}{l}\mathrm{T}_{1}+\text { soil application of borax @ } \\
2.5 \mathrm{~kg} \mathrm{ha}^{-1}\end{array}$ & 93.16 & 13.26 & 44.16 & 12.80 & 23.21 \\
\hline $\mathrm{T}_{5}$ & $\begin{array}{l}\mathrm{T}_{1}+\text { soil application of borax @ } \\
5.0 \mathrm{~kg} \mathrm{ha}^{-1}\end{array}$ & 95.60 & 13.73 & 46.63 & 13.70 & 24.00 \\
\hline $\mathrm{T}_{6}$ & $\begin{array}{l}\mathrm{T}_{1}+\text { soil application of zinc } \\
\text { sulphate @ } 10 \mathrm{~kg} \mathrm{ha}^{-1}+\text { borax @ } \\
2.5 \mathrm{~kg} \mathrm{ha}^{-1}\end{array}$ & 98.56 & 15.46 & 49.20 & 13.96 & 26.73 \\
\hline $\mathrm{T}_{7}$ & $\begin{array}{l}\mathrm{T}_{1}+\text { soil application of zinc } \\
\text { sulphate @ } 10 \mathrm{~kg} \mathrm{ha}^{-1}+\text { borax @ } \\
5.0 \mathrm{~kg} \mathrm{ha}^{-1}\end{array}$ & 101.13 & 17.26 & 52.10 & 14.30 & 26.96 \\
\hline $\mathrm{T}_{8}$ & $\begin{array}{l}\mathrm{T}_{1}+\text { soil application of zinc } \\
\text { sulphate @ } 20 \mathrm{~kg} \mathrm{ha}^{-1}+\text { borax @ } \\
2.5 \mathrm{~kg} \mathrm{ha}^{-1}\end{array}$ & 103.23 & 18.60 & 53.96 & 14.83 & 27.18 \\
\hline $\mathrm{T}_{9}$ & $\begin{array}{l}\mathrm{T}_{1}+\text { soil application of zinc } \\
\text { sulphate @ } 20 \mathrm{~kg} \mathrm{ha}^{-1}+\text { borax @ } \\
5.0 \mathrm{~kg} \mathrm{ha}^{-1}\end{array}$ & 105.30 & 19.53 & 57.06 & 15.33 & 27.41 \\
\hline $\mathrm{T}_{10}$ & $\begin{array}{l}\mathrm{T}_{1}+\text { foliar spray of zinc sulphate } \\
\text { @ } 0.5 \%\end{array}$ & 97.26 & 15.76 & 48.26 & 14.50 & 23.30 \\
\hline $\mathrm{T}_{11}$ & $\begin{array}{l}\mathrm{T}_{1}+\text { foliar spray of solubor @ } \\
0.1 \%\end{array}$ & 94.46 & 14.13 & 47.06 & 15.03 & 24.95 \\
\hline $\mathrm{T}_{12}$ & $\begin{array}{l}\mathrm{T}_{1}+\text { foliar spray of solubor @ } \\
0.25 \%\end{array}$ & 95.10 & 14.50 & 48.03 & 15.83 & 25.83 \\
\hline $\mathrm{T}_{13}$ & $\begin{array}{l}\mathrm{T}_{1}+\text { foliar spray of zinc sulphate } \\
\text { @ } 0.5 \%+\text { solubor @ } 0.1 \%\end{array}$ & 107.13 & 19.23 & 57.46 & 16.73 & 27.84 \\
\hline $\mathrm{T}_{14}$ & $\begin{array}{l}\mathrm{T}_{1}+\text { foliar spray of zinc sulphate } \\
\text { @ } 0.5 \% \text { + solubor @ } 0.25 \%\end{array}$ & 108.80 & 20.60 & 59.70 & 17.43 & 28.68 \\
\hline & SE.m \pm & 2.62 & 0.48 & 1.46 & 0.44 & 1.54 \\
\hline & CD@5\% & 7.61 & 1.41 & 4.26 & 1.30 & 4.49 \\
\hline
\end{tabular}


Table.3 Effect of different methods and levels of zinc and boron application on test weight, number of grains per panicle, number of filled grains per panicle, grain and straw yield

\begin{tabular}{|c|c|c|c|c|c|c|}
\hline $\begin{array}{l}\text { Sl. } \\
\text { No }\end{array}$ & Treatments & $\begin{array}{l}\text { Test } \\
\text { weight } \\
\text { (g) }\end{array}$ & $\begin{array}{c}\text { Number } \\
\text { of } \\
\text { grains } \\
\text { per } \\
\text { panicle }\end{array}$ & $\begin{array}{l}\text { Number } \\
\text { of filled } \\
\text { grains per } \\
\text { panicle }\end{array}$ & $\begin{array}{c}\text { Grain } \\
\text { yield } \\
\left(\mathbf{q} \text { ha }^{-1}\right)\end{array}$ & $\begin{array}{c}\text { Straw } \\
\text { yield } \\
\left(\mathbf{q} \text { ha }^{-1}\right)\end{array}$ \\
\hline $\mathrm{T}_{1}$ & $\mathrm{RDF}+\mathrm{FYM}$ & 25.24 & 96.86 & 74.53 & 48.78 & 61.86 \\
\hline $\mathrm{T}_{2}$ & $\begin{array}{l}\mathrm{T}_{1}+\text { soil application of zinc } \\
\text { sulphate @ } 10 \mathrm{~kg} \mathrm{ha}^{-1}\end{array}$ & 26.25 & 101.87 & 82.46 & 50.33 & 65.03 \\
\hline$T_{3}$ & $\begin{array}{l}\mathrm{T}_{1}+\text { soil application of zinc } \\
\text { sulphate @ } 20 \mathrm{~kg} \mathrm{ha}^{-1}\end{array}$ & 26.86 & 104.00 & 83.20 & 52.50 & 68.31 \\
\hline $\mathrm{T}_{4}$ & $\begin{array}{l}\mathrm{T}_{1}+\text { soil application of borax @ } \\
2.5 \mathrm{~kg} \mathrm{ha}^{-1}\end{array}$ & 27.81 & 107.36 & 87.26 & 56.82 & 74.70 \\
\hline $\mathrm{T}_{5}$ & $\begin{array}{l}\mathrm{T}_{1}+\text { soil application of borax @ } \\
5.0 \mathrm{~kg} \mathrm{ha}^{-1}\end{array}$ & 28.12 & 108.83 & 89.86 & 58.43 & 78.03 \\
\hline $\mathrm{T}_{6}$ & $\begin{array}{l}\mathrm{T}_{1}+\text { soil application of zinc } \\
\text { sulphate @ } 10 \mathrm{~kg} \mathrm{ha}^{-1}+\text { borax @ } \\
2.5 \mathrm{~kg} \mathrm{ha}^{-1}\end{array}$ & 28.88 & 109.26 & 93.33 & 59.86 & 80.30 \\
\hline $\mathrm{T}_{7}$ & $\begin{array}{l}\mathrm{T}_{1}+\text { soil application of zinc } \\
\text { sulphate@10 } \mathrm{kg} \mathrm{ha}^{-1}+\text { borax @ } \\
5.0 \mathrm{~kg} \mathrm{ha}^{-1}\end{array}$ & 29.12 & 111.86 & 95.33 & 60.42 & 81.61 \\
\hline $\mathrm{T}_{8}$ & $\begin{array}{l}\mathrm{T}_{1}+\text { soil application of zinc } \\
\text { sulphate@ } 20 \mathrm{~kg} \mathrm{ha}^{-1}+\text { borax @ } \\
2.5 \mathrm{~kg} \mathrm{ha}^{-1}\end{array}$ & 29.45 & 113.96 & 97.46 & 60.89 & 82.85 \\
\hline $\mathrm{T}_{9}$ & $\begin{array}{l}\mathrm{T}_{1}+\text { soil application of zinc } \\
\text { sulphate@ } 20 \mathrm{~kg} \mathrm{ha}^{-1}+\text { borax @ } \\
5.0 \mathrm{~kg} \mathrm{ha}^{-1}\end{array}$ & 29.85 & 119.43 & 99.70 & 61.48 & 84.93 \\
\hline $\mathrm{T}_{10}$ & $\begin{array}{l}T_{1}+\text { foliar spray of zinc sulphate } \\
\text { @ } 0.5 \%\end{array}$ & 27.13 & 105.30 & 85.00 & 54.06 & 70.61 \\
\hline $\mathrm{T}_{11}$ & $\begin{array}{l}\mathrm{T}_{1}+\text { foliar spray of solubor @ } \\
0.1 \%\end{array}$ & 27.34 & 115.33 & 90.66 & 55.11 & 71.86 \\
\hline $\mathrm{T}_{12}$ & $\begin{array}{l}\mathrm{T}_{1}+\text { foliar spray of solubor @ } \\
0.25 \%\end{array}$ & 27.85 & 116.76 & 92.06 & 56.06 & 73.58 \\
\hline $\mathrm{T}_{13}$ & $\begin{array}{l}\mathrm{T}_{1}+\text { foliar spray of zinc sulphate } \\
\text { @ } 0.5 \%+\text { solubor @ } 0.1 \%\end{array}$ & 31.73 & 119.83 & 102.06 & 62.15 & 86.34 \\
\hline $\mathrm{T}_{14}$ & $\begin{array}{l}T_{1}+\text { foliar spray of zinc sulphate } \\
@ 0.5 \%+\text { solubor @ } 0.25 \%\end{array}$ & 32.47 & 120.70 & 103.50 & 64.40 & 89.40 \\
\hline \multicolumn{2}{|c|}{ SE. $m \pm$} & 0.91 & 0.91 & 2.32 & 1.84 & 1.76 \\
\hline & CD@ $@ 5 \%$ & 2.65 & 2.65 & 6.76 & 5.36 & 5.13 \\
\hline
\end{tabular}


Table.4 Effect of different methods and levels of zinc and boron application on N, P and K content of rice under acid soil

\begin{tabular}{|c|c|c|c|c|c|c|}
\hline \multirow[t]{2}{*}{ Treatments } & \multicolumn{2}{|c|}{$\mathbf{N}$} & \multicolumn{2}{|c|}{$\mathbf{P}$} & \multicolumn{2}{|c|}{$\mathbf{K}$} \\
\hline & Straw & Grain & Straw & Grain & Straw & Grain \\
\hline T1: RDF+FYM & 0.94 & 1.04 & 0.20 & 0.34 & 0.42 & 0.34 \\
\hline $\begin{array}{l}\text { T2: T1+ Soil application of Zinc } \\
\text { Sulpahte @ } 10 \mathrm{~kg} / \mathrm{ha}\end{array}$ & 1.00 & 1.11 & 0.23 & 0.38 & 0.51 & 0.42 \\
\hline $\begin{array}{l}\text { T3: T1+Soil application of Zinc } \\
\text { Sulpahte @ } 20 \mathrm{~kg} / \mathrm{ha}\end{array}$ & 1.05 & 1.17 & 0.22 & 0.36 & 0.56 & 0.47 \\
\hline $\begin{array}{l}\text { T4: T1+ soil application of } \\
\text { borax@ } 2.5 \mathrm{~kg} / \mathrm{ha}\end{array}$ & 0.97 & 1.07 & 0.25 & 0.39 & 0.46 & 0.37 \\
\hline $\begin{array}{l}\text { T5: T1+ soil application of borax @ } \\
5.0 \mathrm{~kg} / \mathrm{ha}\end{array}$ & 1.02 & 1.12 & 0.28 & 0.43 & 0.50 & 0.41 \\
\hline $\begin{array}{l}\text { T6: T1+ Soil application of Zinc } \\
\text { Sulpahte @ } 10 \mathrm{~kg} / \mathrm{ha}+\text { Soil } \\
\text { application of Borax @ } 2.5 \mathrm{~kg} / \mathrm{ha}\end{array}$ & 1.04 & 1.14 & 030 & 0.45 & 0.54 & 0.44 \\
\hline $\begin{array}{l}\text { 77: T1+ Soil application of Zinc } \\
\text { Sulpahte @ } 10 \mathrm{~kg} / \mathrm{ha}+\text { Soil } \\
\text { application of Borax } @ 5.0 \mathrm{~kg} / \mathrm{ha}\end{array}$ & 1.08 & 1.20 & 0.33 & 0.48 & 0.58 & 0.47 \\
\hline $\begin{array}{l}\text { T8: T1+ Soil application of Zinc } \\
\text { Sulpahte @20 kg/ha }+ \text { Soil } \\
\text { application of Borax @ } 2.5 \mathrm{~kg} / \mathrm{ha}\end{array}$ & 1.10 & 1.23 & 0.31 & 0.44 & 0.60 & 0.49 \\
\hline $\begin{array}{l}\text { T9: T1+ Soil application of Zinc } \\
\text { Sulpahte @20 kg/ha + Soil } \\
\text { application of Borax @ } 5.0 \mathrm{~kg} / \mathrm{ha}\end{array}$ & 1.15 & 1.29 & 0.34 & 0.49 & 0.62 & 0.51 \\
\hline $\begin{array}{l}\text { T10: T1+ foliar spray of Zinc } \\
\text { sulphate @ } 0.5 \%\end{array}$ & 1.13 & 1.25 & 0.22 & 0.36 & 0.62 & 0.45 \\
\hline $\begin{array}{l}\text { T11: T1+Foliar spray of solubor @ } \\
0.1 \%\end{array}$ & 0.98 & 1.09 & 0.24 & 0.38 & 0.52 & 0.38 \\
\hline $\begin{array}{l}\text { T12: T1+ Foliar spray of Solubor @ } \\
0.25 \%\end{array}$ & 1.00 & 1.11 & 0.26 & 0.42 & 0.47 & 0.40 \\
\hline $\begin{array}{l}\text { T13: T1+ foliar spray of Zinc } \\
\text { sulphate @ } 0.5 \%+\text { solubor @ } 0.1 \%\end{array}$ & 1.18 & 1.34 & 0.29 & 0.44 & 0.59 & 0.49 \\
\hline $\begin{array}{l}\text { T14: T1+ foliar spray of Zinc } \\
\text { sulphate @ } 0.5 \%+\text { solubor @ } 0.25 \%\end{array}$ & 1.21 & 1.37 & 0.31 & 0.46 & 0.61 & 0.50 \\
\hline SEm \pm & 0.01 & 0.01 & 0.006 & 0.006 & 0.01 & 0.007 \\
\hline CD @5\% & 0.03 & 0.03 & 0.017 & 0.017 & 0.03 & 0.02 \\
\hline
\end{tabular}


Table.5 Effect of different methods and levels of zinc and boron application on $\mathrm{Ca}, \mathrm{Mg}$ and $\mathrm{S}$ content of rice under acid soil

\begin{tabular}{|c|c|c|c|c|c|c|}
\hline \multirow[t]{2}{*}{ Treatments } & \multicolumn{2}{|c|}{ Ca } & \multicolumn{2}{|c|}{ Mg } & \multicolumn{2}{|r|}{$\mathbf{S}$} \\
\hline & Straw & Grain & Straw & Grain & Straw & Grain \\
\hline T1: RDF+FYM & 0.56 & 0.24 & 0.18 & 0.12 & 0.12 & 0.18 \\
\hline $\begin{array}{l}\text { T2: T1+ Soil application of Zinc } \\
\text { Sulpahte @ } 10 \mathrm{~kg} / \mathrm{ha}\end{array}$ & 0.64 & 0.31 & 0.22 & 0.15 & 0.14 & 0.21 \\
\hline $\begin{array}{l}\text { T3: T1+Soil application of Zinc } \\
\text { Sulpahte @ } 20 \mathrm{~kg} / \mathrm{ha}\end{array}$ & 0.68 & 0.36 & 0.24 & 0.19 & 0.17 & 0.24 \\
\hline $\begin{array}{l}\text { T4: } \mathrm{T} 1+\text { soil application of } \\
\text { borax@ } 2.5 \mathrm{~kg} / \mathrm{ha}\end{array}$ & 0.66 & 0.32 & 0.21 & 0.16 & 0.11 & 0.19 \\
\hline $\begin{array}{l}\text { T5: T1+ soil application of borax @ } \\
5.0 \mathrm{~kg} / \mathrm{ha}\end{array}$ & 0.69 & 0.35 & 0.25 & 0.18 & 0.15 & 0.22 \\
\hline $\begin{array}{l}\text { T6: T1+ Soil application of Zinc } \\
\text { Sulpahte @ } 10 \mathrm{~kg} / \mathrm{ha}+\text { Soil } \\
\text { application of Borax @ } 2.5 \mathrm{~kg} / \mathrm{ha}\end{array}$ & 0.72 & 0.39 & 0.27 & 0.20 & 0.16 & 0.24 \\
\hline $\begin{array}{l}\text { T7: T1+ Soil application of Zinc } \\
\text { Sulpahte @ } 10 \mathrm{~kg} / \mathrm{ha}+\text { Soil } \\
\text { application of Borax @ } 5.0 \mathrm{~kg} / \mathrm{ha}\end{array}$ & 0.73 & 0.42 & 0.28 & 0.22 & 0.18 & 0.26 \\
\hline $\begin{array}{l}\text { T8: T1+ Soil application of Zinc } \\
\text { Sulpahte @ } 20 \mathrm{~kg} / \mathrm{ha}+\text { Soil } \\
\text { application of Borax @ } 2.5 \mathrm{~kg} / \mathrm{ha}\end{array}$ & 0.75 & 0.44 & 0.30 & 0.24 & 0.19 & 0.25 \\
\hline $\begin{array}{l}\text { T9: T1+ Soil application of Zinc } \\
\text { Sulpahte @ } 20 \mathrm{~kg} / \mathrm{ha}+\text { Soil } \\
\text { application of Borax @ } 5.0 \mathrm{~kg} / \mathrm{ha}\end{array}$ & 0.78 & 0.48 & 0.32 & 0.27 & 0.21 & 0.27 \\
\hline $\begin{array}{l}\text { T10: T1+ foliar spray of Zinc } \\
\text { sulphate @ } 0.5 \%\end{array}$ & 0.63 & 0.30 & 0.20 & 0.14 & 0.20 & 0.26 \\
\hline $\begin{array}{l}\text { T11: T1+Foliar spray of solubor @ } \\
0.1 \%\end{array}$ & 0.67 & 0.31 & 0.23 & 0.16 & 0.11 & 0.20 \\
\hline $\begin{array}{l}\text { T12: T1+ Foliar spray of Solubor @ } \\
0.25 \%\end{array}$ & 0.69 & 0.34 & 0.24 & 0.17 & 0.13 & 0.22 \\
\hline $\begin{array}{l}\text { T13: T1+ foliar spray of Zinc } \\
\text { sulphate @ } 0.5 \%+\text { solubor @ } 0.1 \%\end{array}$ & 0.83 & 0.52 & 0.35 & 0.29 & 0.24 & 0.28 \\
\hline $\begin{array}{l}\text { T14: T1+ foliar spray of Zinc } \\
\text { sulphate @ } 0.5 \%+\text { solubor @ } \\
0.25 \%\end{array}$ & 0.85 & 0.53 & 0.36 & 0.30 & 0.25 & 0.30 \\
\hline SEm \pm & 0.008 & 0.006 & 0.005 & 0.004 & 0.008 & 0.01 \\
\hline CD @5\% & 0.02 & 0.01 & 0.01 & 0.01 & 0.02 & 0.02 \\
\hline
\end{tabular}


Table.6 Effect of different methods and levels of zinc and boron application on $\mathrm{Zn}$ and B content of rice under acid soil

\begin{tabular}{|c|c|c|c|c|}
\hline \multirow[t]{2}{*}{ Treatments } & \multicolumn{2}{|c|}{$\mathbf{Z n}$} & \multicolumn{2}{|c|}{ B } \\
\hline & Straw & Grain & Straw & Grain \\
\hline T1: RDF+FYM & 25.04 & 21.50 & 1.15 & 1.89 \\
\hline $\begin{array}{l}\text { T2: T1+ Soil application of Zinc Sulpahte @ } 10 \\
\text { kg/ha }\end{array}$ & 31.22 & 25.31 & 1.22 & 2.01 \\
\hline $\begin{array}{l}\text { T3:T1+Soil application of Zinc Sulpahte @20 } \\
\mathrm{kg} / \mathrm{ha}\end{array}$ & 34.49 & 27.14 & 1.34 & 2.14 \\
\hline T4: T1+ soil application of borax@ $2.5 \mathrm{~kg} / \mathrm{ha}$ & 28.84 & 22.82 & 1.55 & 2.54 \\
\hline T5: T1+ soil application of borax @ $5.0 \mathrm{~kg} / \mathrm{ha}$ & 30.12 & 24.68 & 1.62 & 2.87 \\
\hline $\begin{array}{l}\text { T6: T1+ Soil application of Zinc Sulpahte @ } 10 \\
\text { kg/ha + Soil application of Borax @ } 2.5 \mathrm{~kg} / \mathrm{ha}\end{array}$ & 29.00 & 23.22 & 1.70 & 2.66 \\
\hline $\begin{array}{l}\text { T7:T1+ Soil application of Zinc Sulpahte @10 } \\
\mathrm{kg} / \mathrm{ha}+\text { Soil application of Borax @ } 5.0 \mathrm{~kg} / \mathrm{ha}\end{array}$ & 32.59 & 26.39 & 1.75 & 2.73 \\
\hline $\begin{array}{l}\text { T8:T1+ Soil application of Zinc Sulpahte @20 } \\
\mathrm{kg} / \mathrm{ha}+\text { Soil application of Borax @ } 2.5 \mathrm{~kg} / \mathrm{ha}\end{array}$ & 36.87 & 29.53 & 1.78 & 3.05 \\
\hline $\begin{array}{l}\text { T9:T1+ Soil application of Zinc Sulpahte @20 } \\
\mathrm{kg} / \mathrm{ha}+\text { Soil application of Borax @ } 5.0 \mathrm{~kg} / \mathrm{ha}\end{array}$ & 40.19 & 33.20 & 1.83 & 3.13 \\
\hline T10: T1+ foliar spray of Zinc sulphate @ $0.5 \%$ & 41.81 & 34.11 & 1.40 & 2.18 \\
\hline T11: T1+Foliar spray of solubor @ $0.1 \%$ & 28.97 & 23.20 & 1.76 & 2.97 \\
\hline T12: T1+ Foliar spray of Solubor @ $0.25 \%$ & 31.74 & 25.61 & 1.88 & 3.15 \\
\hline $\begin{array}{l}\text { T13:T1+ foliar spray of Zinc sulphate@ } 0.5 \%+ \\
\text { solubor @ } 0.1 \%\end{array}$ & 42.38 & 35.02 & 2.00 & 3.27 \\
\hline $\begin{array}{l}\text { T14:T1+ foliar spray of Zinc sulphate @ } 0.5 \%+ \\
\text { solubor @ } 0.25 \%\end{array}$ & 44.59 & 37.13 & 2.15 & 3.41 \\
\hline SEm \pm & 0.71 & 0.84 & 0.01 & 0.01 \\
\hline CD@5\% & 2.06 & 2.44 & 0.01 & 0.04 \\
\hline
\end{tabular}

The increase in yield attributes with foliar application of zinc and boron might up surge cofactors for enzymatic activation. Consequently, photosynthesis might have enhanced in plants.

Thereafter, higher translocation of carbohydrates to grains, which ultimately resulted in higher grain yield under foliar application of micronutrients. Increase in panicle length and 1000 grain weight by the foliar applied $\mathrm{Zn}$ and $\mathrm{B}$ mixture might be due to higher CGR. It is the fact that B application enhanced the pollen tube germination, grain setting which is involved in metabolism, increased root growth, synthesis of proteins and carbohydrates (Moeinian et al., 2011) which improved the grain yield due by the application of $\mathrm{B}$ and other micronutrients. This is in conformity with Wasaya (2017) reported that foliar application of $\mathrm{Zn}$ and $\mathrm{B}$ in combination has harvested 12 per cent and 45 per cent more yield compared with seed priming and control treatments, respectively. 
Effect of different levels and methods of zinc and boron application on nutrient content and uptake by rice

\section{Major Nutrient Content in straw and grain of rice: $(\mathrm{N}, \mathrm{P}, \mathrm{K}, \mathrm{Ca} \mathrm{Mg}$ and $\mathrm{S})$}

The nutrient content in rice differs significantly due to soil and foliar application of zinc and boron along with NPK+FYM. Significantly higher nitrogen $(1.21 \& 1.37 \%)$, phosphorus $(0.34 \%$ \& $0.49 \%)$, potassium (0.62 and $0.51 \%)$, calcium $(0.85 \& 0.53 \%)$ magnesium $(0.24 \& 0.30 \%)$ and sulphur $(0.25$ $\& 0.30 \%)$ in grain and straw respectively. The lowest nutrient content in grain and straw was recorded in RDF + FYM.

Significant increase in nitrogen, Phosphorus, potassium, calcium, magnesium and Sulphur in straw and grain of rice due to foliar spray of Zinc and boron was attributed to synergistic effect on nitrogen uptake by the crop. These results were in agreement with findings of Aref (2011). Increase in concentration of $\mathrm{Ca}, \mathrm{Mg}$ and $\mathrm{S}$ in plants was due to foliar application of zinc and boron enhanced root growth and enzymatic activity of plant which in turn increased uptake of other elements like $\mathrm{Ca} \mathrm{Mg}$ and $\mathrm{S}$. The $\mathrm{S}$ content in general increased due to foliar spray of Zinc sulphate which contains $\mathrm{S}$ contributed for increase in s content in straw and grain of rice.

\section{Micronutrient content in straw and grain of rice}

The concentration of micronutrients differs significantly due to different treatments. However, significantly higher concentration of zinc $(44.59 \& 37.13 \mathrm{mg} / \mathrm{kg})$ and boron $(2.15 \& 3.41 \mathrm{mg} / \mathrm{kg})$ in straw and grain at harvest significantly increased due to foliar application of zinc and boron @ 0.5 percent and 0.25 percent respectively. Lowest concentration of zinc $(25.04 \& 21.50 \mathrm{mg} / \mathrm{kg})$ and boron $(1.15 \& 1.89 \mathrm{mg} / \mathrm{kg})$ recorded in $\mathrm{RDF}+\mathrm{FYM}$ in straw and grain at harvest.

Higher zinc and boron content in grain and straw was attributed to combined foliar spray of $\mathrm{Zn}$ and Boron has increased concentration in plant rather than soil application. Similar results were also recorded by Aref (2012).

\section{References}

Abbas, M., Zahida, T. M., Uddin, R., Sajjid, I. and Akhla Q., 2013, Effect of zinc and boron fertilizers application on some physico-chemical attributes of five rice varieties grown in Agro-ecosystem of Sindh, Pakistan. American Eurasian J. Agril and environ. sci., 13(4): 433-439.

Anjum, S. A., Saleem, M.F., Shahid, M., Shakoor, A., Safeer, M., Khan, I., Farooq, A., Ali, I. and Nazir, U., 2017, Dynamics of Soil and Foliar Applied Boron and Zinc to Improve Maize Productivity and Profitability. Pak. J. Agril. Res., 30(3): 294-302.

Anonymous, 2016a, Annual report 2016-17. Department of agriculture, Cooperation and farmers' welfare, Pp. 3.

Anonymous, 2016b, http://www.mopsi.gov.in/statisticalyear-book-india/2016/177

Arif, M., Shehzad, M. A., Bashir, F., Tasneem, M., Yasin, G. and Iqbal, M., 2012, Boron, zinc and microtone effects on growth, chlorophyll contents and yield attributes in rice (Oryza sativa L.) cultivar. African J. Biotechnol., 11(48): 10851-10858.

Aslam, M., Mahmood, I. H., Qureshi, R. H., Nawaz, S. and Akhtar, J., 2002, Salinity tolerance of rice as affected by boron nutrition. Pak J. Soil Sci., 21: 110-118.

Black C.A. 1965. Method of Soil Analysis PartII. Agronomy monograph NO.9. American Soc. Agron. Madison, 
Wisconsin, P148.

Cakmak. I. 2008. Enrichment of cereal grain with zinc agronomic or genetic biofertification. Pl Sci. 30(2): 1-17.

Chaudhary, S.K., Singh, S. P., Singh, Y. and Dharminder., 2014, Influence of integrated use of fertilizers and manures on SRI grown rice (Oryza sativa) and their residual effect on succeeding wheat (Triticum aestivum L) in calcareous soil. Indian J. Agron., 59(4): 527-533.

Fang, Y. L., Wang, Z., Xin, L., Zaho, X. and $\mathrm{Hu}, \quad$ Q., 2008, Effect of foliar application of zinc, selenium and iron fertilizers on nutrients concentration and yield of rice grain in china. J. Agri. Food Chem., 56: 2079-2084.

Gomez K.A. and Gomez, 1984. Statistical
Procedure for Agric. Res. $2^{\text {nd }}$ Ed John Wiley \& Sons, New York.

Jackson. 1973. Soil chemical Analysis. Prentice Hall of India Pvt. Ltd, New Delhi

Moeinian, M. R., Zargari, K., Hasanpour, J., 2011, Effect of boron foliar spraying application on quality characteristics and growth parameters of wheat grain under drought stress. Amer. Eur. J. Agric. Environ. Sci., 10, 593-599.

Wasaya, A., Shabir, M. S., Hussain, M., Ansar, M., Aziz, A., Hassan, W. and Ahmad, I., 2017, Foliar application of Zinc and Boron improved the productivity and net returns of maize grown under rain fed conditions of Pothwar plateau. J. Soil Sci. Pl. Nutr., 17 (1): 33-45.

\section{How to cite this article:}

Ravikiran, N.R., S. Channakeshava, R. Jayaramaiah and Nataraja, A. 2019. Effect of Different Methods and Levels of Zinc and Boron Application on Growth and Yield of Rice under Acid Soils of Hilly Zone of Karnataka. Int.J.Curr.Microbiol.App.Sci. 8(07): 1798-1808. doi: https://doi.org/10.20546/ijcmas.2019.807.214 